\title{
EXISTENCE OF POSITIVE ENTIRE SOLUTIONS OF A SEMILINEAR $p$-LAPLACIAN PROBLEM WITH A GRADIENT TERM
}

\section{YING SHEN AND JiHUi ZHANG}

Abstract. In this paper, we study a semilinear $p$-Laplacian problem

$$
-\Delta_{p} u+h(x)|\nabla u|^{q}=b(x) g(u), u>0, x \in \mathbb{R}^{N}, \lim _{|x| \rightarrow \infty} u(x)=0,
$$

where $q \in(p-1, p], b, h \in C_{l o c}^{\alpha}\left(\mathbb{R}^{N}\right)$ for some $\alpha \in(0,1), h(x) \geqslant 0, b(x)>0, \forall x \in \mathbb{R}^{N}$, and $g \in C^{1}((0, \infty),(0, \infty))$ which may be singular at 0 . Using a sub-supersolution argument and a perturbed argument, we obtain the existence of entire solutions to the problem. No monotonicity condition is imposed on the functions $g(s)$ and $\frac{g(s)}{s^{p-1}}$.

Mathematics subject classification (2010): 35J61, 35J91.

Keywords and phrases: semilinear $p$-Laplacian problem, entire solutions, existence.

\section{REFERENCES}

[1] H. Brezis, S. Kamin, Sublinear elliptic equations in $\mathbb{R}^{N}$, Manuscripta Math., 74 (1992), 87-106.

[2] H. BREZIS, L. OSWALD, Remarks on sublinear elliptic equations, Nonlinear Anal., 10 (1986), 55-64.

[3] F. CĬRSTEA, V.D. RĂDULESCU, Existence and uniqueness of positive solutions to a semilinear elliptic problem in $\mathbb{R}^{N}$, J. Math. Anal. Appl., 229 (1999), 417-425.

[4] D.-P. COVEI, Existence and asymptotic behavior of positive solution to a quasilinear elliptic problem in $\mathbb{R}^{N}$, Nonlinear Anal., 69 (2008), 2615-2622.

[5] J.I. DIAZ, J.E. SAÀ, Existence et unicite de solutions positives pour certaines equations elliptiques quasilineaires, C. R. Acad. Sci. Paris Sér. I Math., 305 (1987), 521-524.

[6] T.-L. Dinu, Entire solutions of sublinear elliptic equations in anisotropic media, J. Math. Anal. Appl., 322 (2006), 382-392.

[7] A. L. Edelson, Entire solutions of singular elliptic equations, J. Math. Anal. Appl., 139 (1989), 523-532.

[8] W. FENG, X. LIU, Existence of entire solutions of a singular semilinear elliptic problem, Acta Math. Sinica, 20 (2004) 983-988.

[9] D. Gilbarg, N. S. Truginger, Elliptic Partial Differential Equations of Second Order, SpringerBerlin, 1998.

[10] J. V. Goncalves, C. A. SAntos, Positive solutions for a class of quasilinear singular equations, Electron. J. Differential Equations, 56 (2004), 1-15.

[11] J. V. Goncalves, C. A. SAntos, Existence and asymptotic behavior of non-radially symmetric ground states of semilinear singular elliptic equations, Nonlinear Anal., 66 (2007), 2078-2090.

[12] O. A. LAdyzenskaja, N. N. UraL'TSEVA, Linear and Quasilinear Elliptic Equations, Academic Press, 1968.

[13] A. V. LAIR, A. W. ShaKer, Entire solutions of a singular elliptic problem, J. Math. Anal. Appl., 200 (1996), 498-505.

[14] A. V. LAir, A. W. ShaKer, Classical and weak solutions of a singular elliptic problem, J. Math. Anal. Appl., 211 (1997), 371-385.

[15] C. L. LIU, Z. D. YANG, Existence of large solutions for a quasilinear elliptic problem via explosive sub-supersolutions, Appl. Math. Comput., 199 (2008), 414-424. 
[16] H. MAAGLI, M. ZRIBI, Existence and estimates of solutions for singular nonlinear elliptic problems, J. Math. Anal. Appl., 263 (2001), 522-542.

[17] H.T. XUE, X. G. SHAO, Existence of positive entire solutions of a semilinear elliptic problem with a gradient term, Nonlinear Anal., 71 (2009), 3113-3118.

[18] D. YE, F. ZHOU, Invariant criteria for existence of bounded positive solutions, Discrete Contin. Dyn. Syst., 12 (2005), 413-424.

[19] Z. ZHANG, A remark on the existence of entire solutions of a singular elliptic problem, J. Math. Anal. Appl., 215 (1997), 579-582.

[20] Z. ZHANG, A remark on the existence of positive entire solutions of a sublinear elliptic problem, Nonlinear Anal., 67 (2007), 147-153. 